
cultures

Les cahiers de l'Acedle

10-1 | 2013

Apprendre les langues autrement

\title{
Acquisition du français par les Chinois
}

Problématiques liées à l'expression de l'idée de futur

\section{Christine Cuet}

\section{OpenEdition}

Journals

Édition électronique

URL : http://journals.openedition.org/rdlc/1532

DOI : $10.4000 /$ rdlc. 1532

ISSN : 1958-5772

Éditeur

ACEDLE

Référence électronique

Christine Cuet, "Acquisition du français par les Chinois », Recherches en didactique des langues et des cultures [En ligne], 10-1 | 2013, mis en ligne le 07 avril 2017, consulté le 19 avril 2019. URL : http:// journals.openedition.org/rdlc/1532; DOI : 10.4000/rdlc. 1532

Ce document a été généré automatiquement le 19 avril 2019.

\section{(c) $(1)$}

Recherches en didactique des langues et des cultures is licensed under a Creative Commons AttributionNonCommercial-NoDerivatives 4.0 International License 


\title{
Acquisition du français par les Chinois
}

Problématiques liées à l'expression de l'idée de futur

\author{
Christine Cuet
}

Je remercie les professeurs de FLE (Français Langue Étrangère) et de CLE (Chinois Langue Étrangère) qui m'apportent une aide précieuse pour le recueil des données ainsi que pour leurs observations et remarques; en particulier Audrey Marguerie, Shuying Zhang-Marcot, Linda Onu, Sylvie Barrier.

Je remercie aussi les étudiants de Master FLE de l'université de Nantes, Français et Chinois, qui participent à la réflexion, à la constitution et à l'analyse des corpus pendant leurs stages.

Le temps va et vient et vire et moi, las! Ne sais que dire!

(Bernard de Ventadour)

\section{Introduction}

Une partie de mes recherches en didactique du FLE a pour but d'analyser et de comprendre les difficultés à progresser des étudiants chinois de niveau B1/B2 du CECRL (Cadre Européen Commun de Référence pour les Langues, 2001), à partir d'erreurs récurrentes translinguistiques et transculturelles. Il s'agit de proposer des hypothèses explicatives qui s'appuient sur l'analyse de productions écrites et orales, de questionnaires, recueillis en France et en Chine dans des cours de FLE, ainsi que sur des entretiens avec des étudiants de Master FLE dont je dirige les mémoires de fin d'études. Je présente ici la synthèse de plusieurs travaux, dans une perspective de recherche-action pour une didactique contextualisée.

Dans les parties 2 et 5 , je montre que l'enseignement traditionnel de la grammaire et les manuels sont en partie à l'origine des problèmes rencontrés; dans les parties 3 et 4 j'explique mes hypothèses concernant la représentation du temps et son expression linguistique, à la source de nombreuses erreurs ; dans la partie 6, je présente le résultat de 
l'analyse du corpus écrit portant sur l'expression du futur, qu'il soit objectivement proche ou lointain, qu'il soit certain, probable ou hypothétique.

\section{L'enseignement de la grammaire du français en Chine}

4 Les ouvrages écrits par les Chinois sur leur langue portent, dès les temps anciens, sur la lexicographie et la dialectologie, car la tradition chinoise ne propose pas de description grammaticale. C'est seulement au XIXe s. que la pression culturelle de l'occident impulse les premières études de grammaire générale, sur des bases contrastives, les ouvrages publiés auparavant étant ceux de missionnaires jésuites œuvrant pour l'expansion du christianisme, manuels et recueil de règles, à diffusion très limitée. Il en découle une représentation d'absence de grammaire, renforcée par l'absence de marques flexionnelles (genre, nombre, conjugaisons, etc.) ; beaucoup d'étudiants chinois pensent que leur langue est sans grammaire puisque la plupart des langues diffusées en Chine sont flexionnelles (langues indo-européennes dont l'anglais obligatoire) et que leur formation de base linguistique porte presque exclusivement sur l'apprentissage de la lecture/ écriture des sinogrammes.

De plus, les étudiants sont formatés dès le secondaire quand ils apprennent l'anglais. Mes précédentes enquêtes (Cuet, 2011) montrent en effet l'existence d'une représentation largement partagée que les listes de vocabulaire et les règles de grammaire à apprendre par cœur sont considérées comme "le plus important" pour maîtriser le français et "réussir les examens" qui portent sur l'écrit dans les départements de français des universités. Beaucoup pensent que la mise en pratique de leurs connaissances dans les cours de communication/conversation (proposés par les lecteurs français) est souvent une "perte de temps", d'autant plus qu'ils ne sont pas habitués à prendre la parole en classe, à exprimer une opinion, dans une société où la pensée collective est de règle et où le rôle du professeur est de transmettre "clairement" les savoirs à apprendre, autrement dit ce qu'il faut mémoriser et reproduire. La communication interculturelle est souvent considérée comme secondaire par rapport à l'apprentissage linguistique.

Quand on consulte les manuels publiés en Chine les plus utilisés à l'université, comme Le français de Ma Xiaohong (1992) ${ }^{1}$, en perpétuelle réédition, on constate que la grammaire enseignée est traditionnelle (Grevisse, Larousse, Bescherelle ou Le Petit Robert pour la prononciation des mots isolé, etc.), avec la terminologie métalinguistique à apprendre parallèlement, alors qu'elle est mal adaptée à la description de la langue chinoise, pour la comparaison interlinguistique. Par exemple (Roche, 2007), un mot chinois comme 工作 gōng zuò peut être traduit en français par un verbe "travailler" ou un nom "travail", en fonction de sa place dans la phrase chinoise ; comme il n'y a ni conjugaison, ni genre et nombre en chinois, c'est le contexte et les autres éléments de la phrase qui permettent de décider de la classe grammaticale de ce mot, nom ou verbe.

7 L'anglais, première langue indo-européenne apprise, à tendance isolante, est souvent considéré comme moins étrange que le français, d'autant plus qu'il y a beaucoup de noms/verbes ayant la même forme non marquée comme en chinois, en raison d'une morphologie simplifiée. Ainsi, "dance" est nom/verbe en anglais quand le français distingue les mots "danse" (nom) et "danser" (verbe). 


\section{Représentations sur le temps}

8 La métaphore universelle "time is moving" (Lakoff \& Johnson, 1985) est soumise à des variations en fonction des langues et des cultures : le temps, exprimé en termes spatiaux à partir de l'expérience humaine, est représenté différemment en chinois et en français (Ahrens \& Chu-Ren Huang, 2002 ; Cuet \& al., 2008 ; Gentner \& al., 2002 ; Boroditsky \& al., 2010).

\section{Kronos et Kairos}

9 Le temps objectif mesurable (Kronos) s'écoule du passé vers le futur (time-moving metaphor). En revanche le temps subjectif s'écoule du passé s'éloignant vers le futur qui s'approche, par rapport à l'homme ancré et agissant dans son présent (Kairos), situé entre passé et futur (ego-moving metaphor) (Jullien, 2001 ; Gentner \& al., 2002). Pour Jullien, il semblerait que le Kairos soit la dimension privilégiée de la culture chinoise. Ce temps pourrait s'inscrire dans la durée subjective du temps qui passe et non dans l'instant fugace objectif du temps qui s'écoule.

\section{Les métaphores}

Gentner \& al. (2002) distingue deux métaphores sur le temps :

Figure 1 - Les métaphores sur le temps (Gentner et al., 2002)

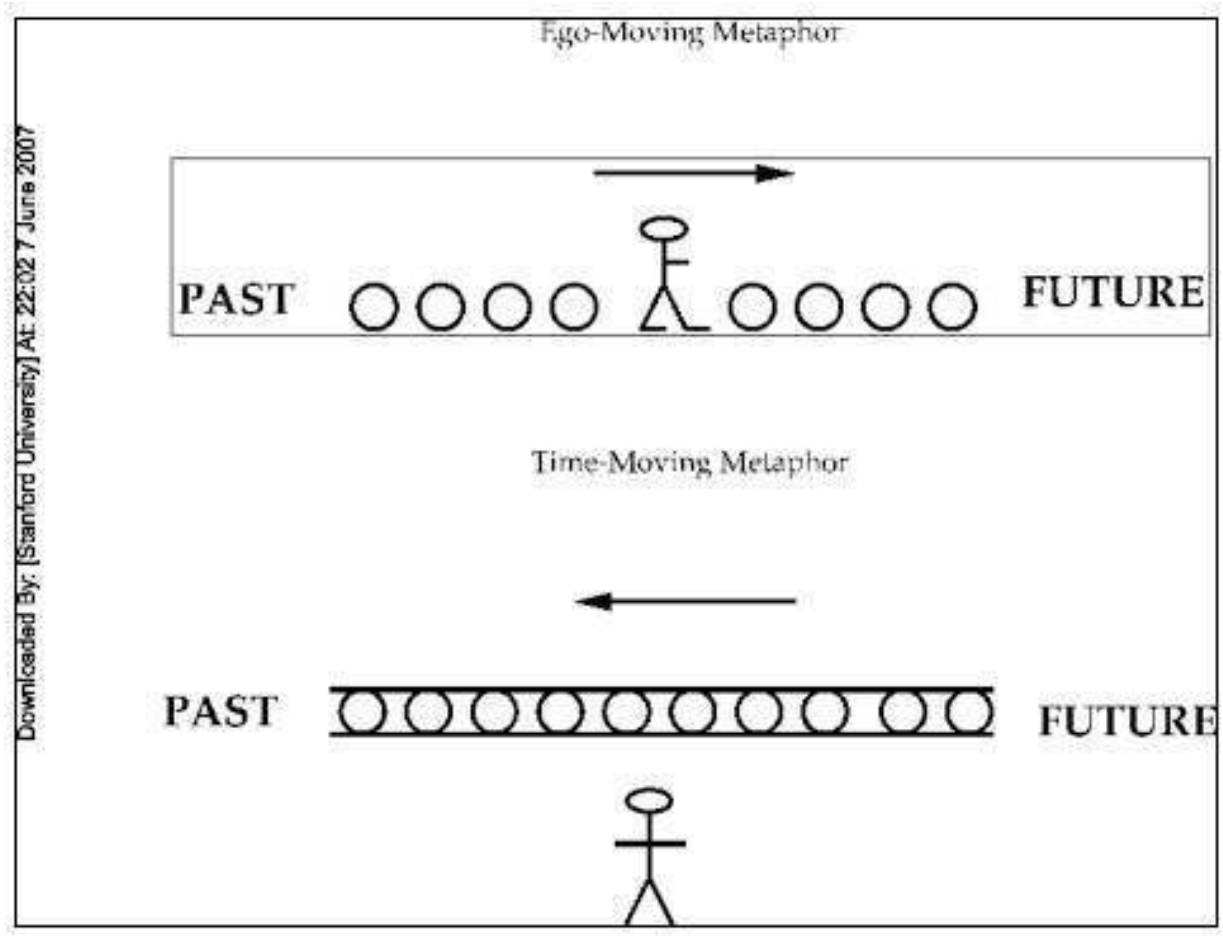




\section{Time moving metaphor}

11 Les représentations linéaires du temps des Chinois comportent souvent un rond ou un intervalle important pour représenter le présent, défini par beaucoup d'étudiants par la notion de "jour présent" impliquant la durée et non "moment/instant présent" qui est la représentation courante des étudiants français.

Figure 2 - représentation de la ligne du temps par une Chinoise

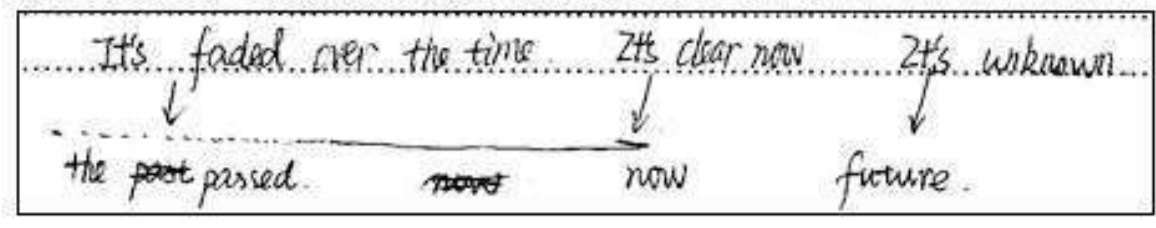

Dans ce dessin réalisé par une étudiante ${ }^{2}$ en Chine, la flèche du temps représente en pointillé le passé qui s'en va et s'estompe, et en ligne pleine le présent qui s'inscrit dans la durée (Kairos). Le mot "now" est barré puis réécrit pour représenter l'instant présent (ce qui modifie la représentation chinoise initiale). Le futur, qui n'existe pas encore, n'est pas représenté sur la ligne du temps, car il est incertain/inconnu. À titre d'exemple, "l'année dernière" 去年 qù nián (partir-année) est symbolisée par un caractère qui signifie l'éloignement par rapport à soi, et "l'année prochaine" 明年 míng nián (lumière - année) est symbolisée par le retour cyclique du soleil ${ }^{3}$.

\section{Ego-moving metaphor}

Plusieurs dessins témoignent d'une différence culturelle d'orientation du regard: l'étudiante française regarde et avance en direction de l'avenir, elle l'exprime sur le plan linguistique par son système verbal et ses métaphores spatiales; le futur se trouve devant elle, le passé derrière elle : "on tourne le dos au passé et on fait face à l'avenir".

Figure 3 - Représentation de la métaphore par une Française

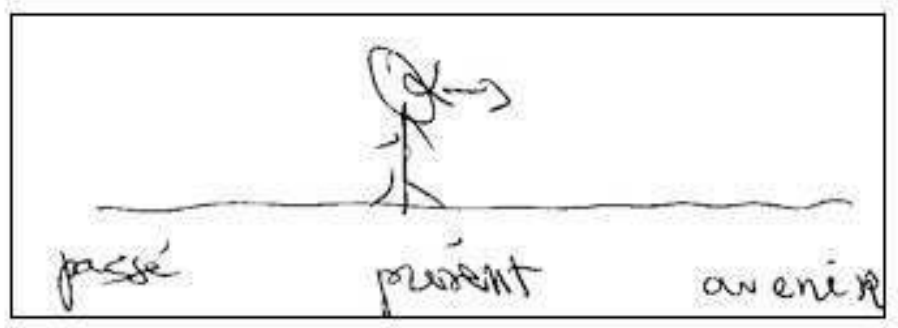

14 À l'inverse, l'étudiante chinoise regarde en direction du passé, car on apprend du passé pour agir dans l'avenir selon la tradition confucéenne. Ce qui est devant soi est connu. En revanche ce qui est derrière soi et qu'on ne voit pas encore est le futur, inconnu même s'il est parfois prévisible ou que plusieurs choix font partie du possible : "je vois le passé, pas l'avenir, il changer". Cette conception du temps n'est pas spécifique à la Chine. Le passé est devant l'homme qui progresse ici vers le futur à reculons, puisqu'il ne peut le représenter. 
Figure 4 - Représentation de la métaphore par une Chinoise

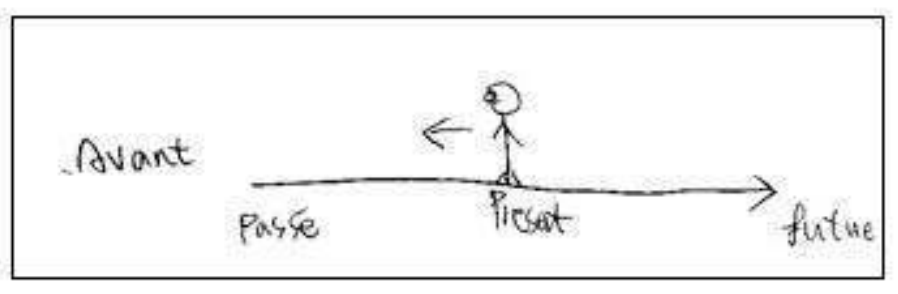

15 Dans la culture chinoise, "le temps et l'espace ne sont pas dissociables l'un de l'autre" (Jullien, 2001). Un seul mot peut exprimer à la fois la référence spatiale ou la référence temporelle. Sur l'axe horizontal, 前 qián "devant" (front) est utilisé pour parler du passé, 后 hòu "derrière" (back) pour parler du futur, par exemple "avant-hier" 前天 qián tiān (devant/avant - jour) et "après-demain" 后天 hòu tiān (derrière/arrière - jour).

16 Il en résulte des problèmes d'acquisition relevés aussi chez des étudiants ayant un bon niveau de français (B1 acquis). Par exemple, dans la phrase "le meilleur est *devant nous", l'étudiante utilise la préposition spatiale et fait référence au passé, alors qu'en français cette phrase fait référence au futur. Un autre exemple est "l'année *derrière", soit celle qui est derrière celle qui s'en va que l'on voit devant soi ( = "l'année dernière" 去年 qù nián), ce qui correspond à "il y a deux ans". Ces erreurs parfois surprenantes, concernant les prépositions locatives de l'espace et du temps que les Chinois ont du mal à s'approprier, sont d'origine culturelle. Elles peuvent passer inaperçues en classe, en l'absence de date ou de contexte approprié et conduire à des quiproquos.

17 Cette double référence spatio-temporelle est aussi inscrite dans les caractères 上 shàng (sur-haut/dernier) et 下 xià (sous-bas/prochain). Ce qui est visible et connu, audessus du plan terrestre, relève du passé ; ce qui est invisible et inconnu, au-dessous du plan terrestre, relève de l'avenir. Ces deux caractères sont également porteurs de valeurs axiologiques positive et négative : selon Confucius, le passé est précieux, d'où également le sens "supérieur" de shàng et le sens "inférieur" de xià". 
Figure 5 - Exemples lexicaux liés à la représentation de la temporalité (Cuet)

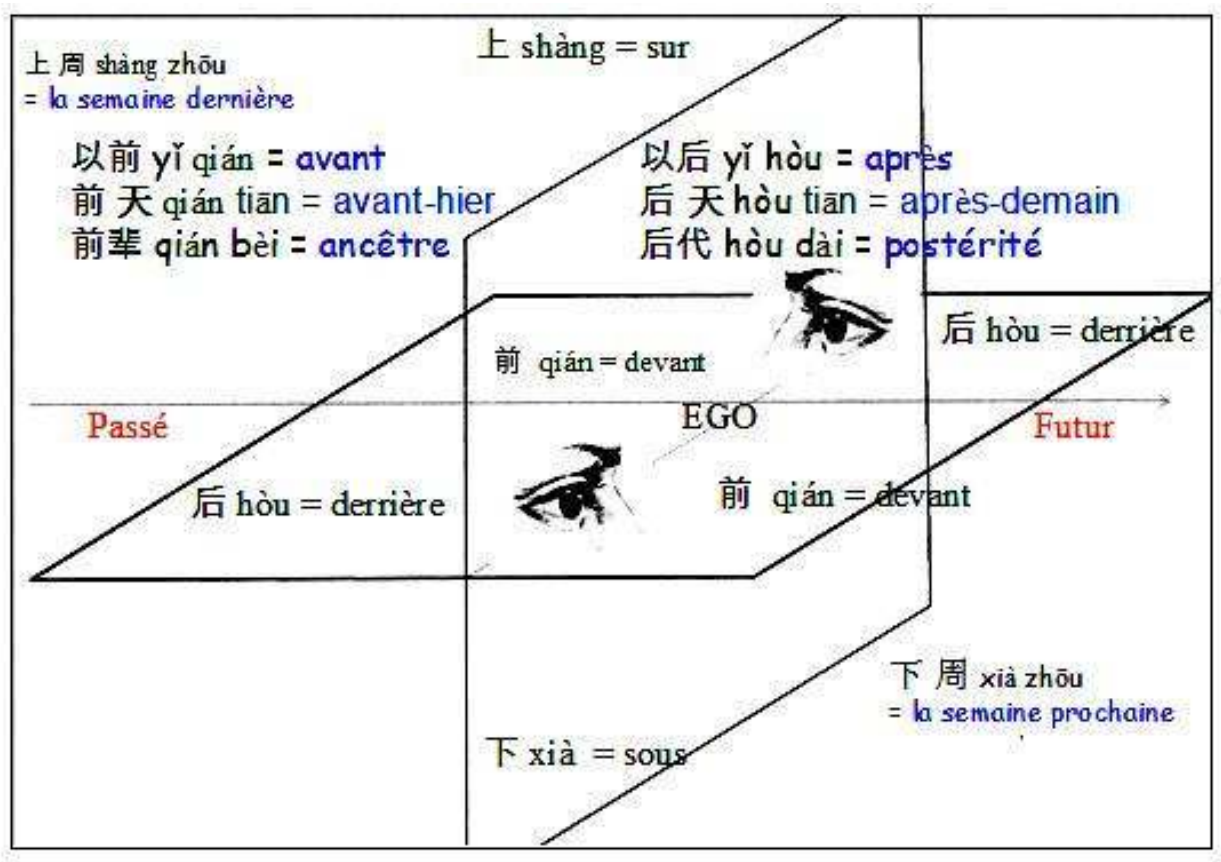

Le schéma de Gentner et al. (Figure 1) est une représentation que je qualifierais "occidentale", le sens de la marche devant être inversé dans la représentation traditionnelle de l'ego-moving metaphor inscrite dans les sinogrammes (marche à reculons). Ce qui n'implique pas, dans la réalité moderne, que les Chinois ne se projettent pas dans l'avenir, surtout à l'ère moderne de la mondialisation (où ils font preuve d'une exceptionnelle faculté d'adaptation) qui induit un changement profond du système de valeurs sur les plans idéologique, économique et social, même si les traditions ainsi que les modes de relation/communication interindividuelle décrits par $\mathrm{Pu}$ (2003) ou Zheng (2002) restent encore fortement ancrés chez une majorité de Chinois, en l'absence de contacts personnels ou professionnels avec des occidentaux.

Cette conceptualisation égocentrique de l'espace métaphorisé pour exprimer le temps a pour cadre cognitif (Levinson, 2003) la référence intrinsèque fondée sur les parties inhérentes de l'objet vu ou représenté (spatial/temporel) et la référence relative fondée sur les axes corporels de l'observateur (horizontalité/verticalité). Les Chinois utilisent aussi le cadre de référence absolu (points abstraits et prédéfinis comme les points cardinaux à la base du plan et de l'architecture "échiquier" des villes ainsi que de l'orientation des maisons traditionnelles et la disposition des pièces); par exemple pour indiquer une direction, la formulation "tu tournes à l'est/ouest" tend actuellement à être remplacé par la latéralité "à main gauche/droite", sans doute à cause de l'influence des langues occidentales ${ }^{5}$.

\section{Expression du futur en chinois}

Sur le plan linguistique, le chinois est une langue isolante, les mots sont invariables. La temporalité et l'aspectualité sont notées par des adverbes et des particules. Par conséquent, les étudiants chinois ont beaucoup de difficultés à assimiler et à manipuler le système verbal du français (conjugaisons et emploi des formes verbales) qu'ils jugent 
beaucoup plus complexe que celui de l'anglais, première langue indo-européenne apprise avant le français.

La comparaison interlinguistique dépasse rarement la question de la morphologie verbale, les étudiants chinois soulignent qu'"en anglais il y a peu de conjugaison", alors que la morphologie française est considérée comme un "casse-tête", et jugée comme "le plus difficile de la grammaire française" par les étudiants débutants, voire intermédiaires (Cuet, 2011). Les apprentissages portent généralement sur la mémorisation des formes linguistiques au détriment de leur usage pragmatique. Dans des classes observées en Chine, où l'alternance codique est courante, les professeurs chinois expliquent en chinois mandarin la grammaire française, passent aussi par l'anglais pour gagner du temps en traduisant ou pour éviter des erreurs d'interprétation. Les productions montrent que l'anglais est à l'origine de nombreux calques sémantiques et syntaxiques (voir infra les corpus).

En chinois, le futur est indiqué par le contexte ou par :

- des adverbes temporels, plus nombreux et plus nuancés qu'en français, par exemple míng tiān "demain", kuài "bientôt" (changement qui va se produire incessamment et se trouve donc généralement associé à $了$ le, particule finale qui marque le changement d'état), mă shàng "tout de suite" qui indique une action imminente, etc. ;

- des verbes modaux : 要 yào qui exprime la volonté du locuteur à réaliser une action, 可以 kĕ yı̆, 会 huì, qui expriment la possibilité (huì pour insister aussi sur la capacité du sujet de réaliser l'événement).

\section{Le verbe 要 yào "vouloir, falloir, aller"}

La phrase "wǒ yào chī fàn" peut être traduite en français par "je veux/je dois manger" ou "je vais manger", en fonction du contexte. Il est possible d'avoir une double interprétation, à la fois modale et temporelle. Si l'on veut marquer le déplacement du sujet énonciateur dans un autre lieu pour réaliser le procès, on ajoutera 去 qù qui signifie "aller vers un autre lieu/s'éloigner du lieu actuel" après yào : "wǒ yào qù chī fàn".

Figure 6 - l'intention

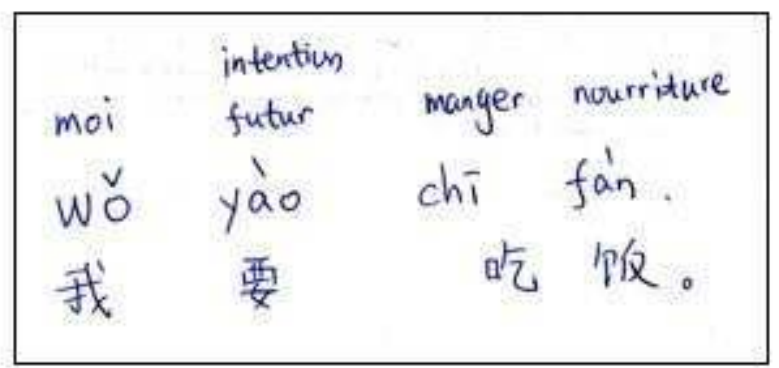

Dans l'exemple oral de description d'itinéraire qui suit, l'étudiant (parlant couramment anglais, en école d'ingénieur en France) hésite entre les deux formes en compétition dans son interlangue (le système linguistique intériorisé et évolutif de la langue-cible) "tu veux" et "tu vas" :

Tu peux partir de la porte de l'université et euh aller au droit euh ensuite tu veux remarquer un grand cross, il y a plusieurs rues euh là-bas tu veux tu vas-tu vas voir un café euh euh donc euh tu prends tu prends la rue à côté du café euh ensuite tu 
euh tu euh aller tu vas au droit sur la rue tu vas voir un immeuble haute et il [blank] il est blanc c'est les courses (=CROUS) du l'université euh l'IUT est juste à côté de le CROUS euh tu veux tu vas trouver IUT sans difficulté.

\section{Le verbe 会 huì "pouvoir" avec les verbes de perception}

En chinois on utilise aussi kě yĭ et huì exprimant la possibilité et la capacité, ils sont traduits en français par le verbe "pouvoir". Dans le corpus oral recueilli en France et dans le corpus écrit chinois traduit en français par un professeur chinois, les étudiants concluent en faisant souvent un transfert du chinois 会 huì et/ou de l'anglais "you can see" :

Tu peux marcher sur la route pour dix minutes ensuite tu peux voir une grande bus station et une blanche maison et devant devant euh devant leur tu peux voir iut c'est bon.

Enfin tu peux voir ma mon ma maison qui s'appelle Place Viarme, tu peux trouver la place faci facilement.

Q 28: au long [yán zhe] de cette route [zhè tiā lù] va vers l'est [xiàng dōng zŏu] et tu va pouvoir [hui] voir [kàn] l'arrêt de bus.

Q.31: ensuite [zài] va vers l'est [wăng dŏng zŏu] et tu vas pouvoir [jiù kě yĭ] arriver [dào...le] à la banque

会 huì note aussi la conséquence d'un autre événement permettant de prévoir le futur (Wu 2010), ce qui explique qu'il est couramment utilisé dans les descriptions d'itinéraires.

\section{L'aspect accompli, la particule 了 le}

En français, on peut utiliser le futur antérieur pour marquer la succession des actions : "quand tu auras traversé le pont, prends la première à droite, c'est par là"; on peut conclure la description d'un itinéraire par un temps du futur : "Descendez la rue jusqu'au rond-point. Tournez à gauche. Sur la droite, vous verrez un fleuriste, c'est un peu plus loin". En chinois, on conclut souvent la description d'un itinéraire par la particule 了 le, qui indique un aspect accompli ou un changement d'état, par exemple :

Q 33 : [jiù dào le] (déjà / aussitôt-justement-arriver / accompli).

Dans les corpus recueillis, même quand il s'agit d'étudiants plus avancés (niveau B1/B2), l'impératif est rarement utilisé, car il est appris pour exprimer un ordre. Le futur n'est pas utilisé car il interfère, sur le plan discursif, avec l'accompli du chinois marquant la fin de l'explication.

\section{Les manuels de FLE}

J'ai choisi ces exemples de descriptions d'itinéraire parce qu'ils apparaissent assez rapidement dans la suite des leçons des manuels français et sont liés à un objectif de survie. Le temps "futur simple" n'est abordé qu'en fin de manuel de niveau A2 et la thématique associée est le plus souvent "décrire ses projets". En l'absence de reprise de "décrire un itinéraire", dans une logique de développement en spirale, les productions des étudiants restent calquées sur le chinois et l'anglais, par exemple l'expression de la distance en durée (discursif - chinois) ou l'utilisation de la préposition "pour" à la place 
de "pendant" (linguistique - traduction de l'anglais), soit un calque des deux langues à la fois dans une phrase comme "tu peux marcher pour ${ }^{6}$ dix minutes". Dans une mégalopole chinoise, en réponse à la question "c'est loin ?", on vous répondra en effet "c'est à 10 minutes", alors qu'en français, on dirait plutôt spontanément "c'est à 200 mètres", sauf dans le cas éventuel d'une demande précise. Les étudiants français consultés poseraient éventuellement cette question à un passant en cas d'hésitation entre faire un trajet à pied ou en transport en commun ou à un chauffeur de bus.

\section{Influence de la méthodologie}

30 Je pense que la conception des manuels utilisés (qu'ils soient français ou locaux) et la méthodologie de l'enseignement influencent fortement l'acquisition. Beaucoup d'erreurs ont pour origine un sur-apprentissage de règles grammaticales qui ne sont pas toujours conformes aux usages actuels du français cultivé; elles sont parfois inadéquates, voire caduques, étant donné l'évolution de la langue. De plus, ces règles sont apprises, dans toute leur complexité, sans liens véritables avec les besoins langagiers communicatifs auxquels seront confrontés les jeunes Chinois qui viennent en France poursuivre leurs études. C'est ce que l'on constate à la lecture de nombreux manuels chinois. D'un autre côté, dans les manuels français à tendance universaliste, les actes de langage sont souvent présentés en termes de progression, avec des thématiques prototypiques spécifiques et peu réutilisées à l'occasion de l'apprentissage d'un nouveau point de grammaire, comme je l'ai montré avec la description d'itinéraire. Les manuels proposent souvent des listes de mots ou d'expressions pour un acte de parole et l'application de règles grammaticales, avec des fiches récapitulatives contenant une multitude d'exercices qui ne facilitent pas l'appropriation parce qu'il n'y a pas beaucoup de remarques explicites concernant ce qu'il faut apprendre et mémoriser en priorité. Les étudiants critiquent souvent nos manuels dont ils ne comprennent pas le déroulement et la logique alors qu'ils sont rassurés par leur mode d'apprentissage par cœur et l'impression de sécurité face aux exercices applicatifs traditionnels. Les exercices de conceptualisation et de créativité ne font pas partie de leur culture éducative (corpus de mémoires d'étudiants chinois de Master 1 portant sur la comparaison des méthodologies et des manuels en France et en Chine (Xu Y, 2010).

\section{Futur et futur proche}

31 La consigne des professeurs chinois est de traduire la forme progressive de l'anglais par la forme périphrastique aller + infinitif du français définie comme un futur proche dans le temps et la forme modale "will" par le temps futur du français, défini comme un futur lointain, un peu plus probable et prédictif que le conditionnel appris en tant qu'expression de la politesse française pour "je voudrais" et ultérieurement dans les manuels pour l'expression du souhait, du conseil, de l'hypothèse ou de la supposition.

Les étudiants chinois, même au niveau B1/B2, ne comprennent pas bien qu'on puisse dire en français "je pars/je vais partir/je partirai demain/l'année prochaine" dans les conversations ordinaires et que cela ne relève pas de la proximité ou de l'éloignement par rapport au temps objectif. Le présent est rarement utilisé puisqu'il y a des formes verbales spécifiques pour le futur, d'autant plus qu'apprendre par cœur les conjugaisons 
a demandé beaucoup d'efforts. Le futur antérieur n'est pas non plus utilisé, car les étudiants ne maitrisent pas la syntaxe des phrases complexes où il est employé.

Les manuels, en proposant des exercices à trous ou des exercices structuraux favorisent le plus souvent le "savoir conjuguer". Il n'y a pas vraiment d'explication pour le choix des formes, sauf la "recette" qu'il est possible d'utiliser le futur périphrastique quand on ne sait pas conjuguer un verbe au futur simple.

\section{Analyse du corpus sur le futur}

34 J'ai sélectionné 10 productions écrites sur un total de 48 (B1/B2 - étudiants en Master ou en école d'ingénieur, tous poursuivant leurs études en France depuis plus de 6 mois). La consigne était d'écrire un texte accompagné d'un dessin : "Raconte comment tu imagines le monde ou ta vie dans 10, 20, 50 ans...". Les textes qui illustrent les résultats sont caractéristiques de l'ensemble du corpus. Les commentaires portant sur l'utilisation des formes verbales ont été rédigés après évaluation préalable des productions sur le plan discursif et linguistique et discussions avec des étudiants chinois et français en formation Master FLE.

\section{Premiers constats et hypothèses explicatives}

Les textes sont très rarement écrits au présent. Plusieurs étudiants chinois interrogés à ce sujet expliquent qu'il y a rupture entre le présent qui concerne le jour/le mois/l'année en cours (ce qui est cyclique) et le futur lointain de la consigne ("dans 10 ans..."). Le présent est le temps qui s'écoule, il possède une densité (+ duratif, cf. 3.2.1. supra) ressentie subjectivement. De plus, pourquoi utiliser le présent puisqu'il y a des "temps" spécifiques? La consigne a souvent été interprétée comme un prétexte pour vérifier leurs connaissances sur la conjugaison malgré l'appel à l'imaginaire et à la multi modalité (texte et dessin - exemple infra en annexe).

Les étudiants adoptent diverses stratégies, liées à leur connaissance de la morphologie verbale et à leur représentation globale de la temporalité en français ${ }^{7}$. La stratégie d'évitement de certains étudiants, pour ne pas faire d'erreurs, consiste à choisir le futur périphrastique appris en premier et "plus facile à conjuguer". D'autres, malgré leur mauvaise connaissance de la conjugaison utilisent quand même le futur simple, conformément à ce qu'ils ont appris sur la différence proche/lointain, en fonction du temps objectif de la projection.

Les textes montrent aussi une méconnaissance sur la façon d'exprimer des souhaits, des hypothèses et des préférences. Le futur simple et le conditionnel à la première personne sont confondus. Après vérification, s'il n'y a pas de -s à "j'aimerai", c'est bien parce qu'il s'agit du futur, ce n'est pas une erreur d'orthographe grammaticale concernant la désinence du conditionnel. Une hypothèse est que la variété du français oral ne permet pas d'ancrer les règles normatives apprises concernant la distinction futur/conditionnel ; par exemple, pour "j'aimerais bien", la prononciation canonique [è] est réalisée par les Français sur un continuum qui va du /e/ fermé, /E/ moyen à /è/ ouvert, ce qui favorise sans doute inconsciemment cette confusion car la distinction phonologique normative apprise en Chine ne coïncide pas forcément avec ce qui est perçu en France, en communication authentique. 


\title{
Exemples du corpus
}

\begin{abstract}
Je travaillerai dans une entreprise française dans 10 ans. Je serai injénieur de mécanique et parfois je traduirai les dossiers français pour mes collègues. Comme c'est une entreprise internationale, je devrai partir beaucoup en mission et je mettrai beaucoup de temps dans l'avion (Raphael).

Après 10 ans, je deviendrai une mère de deux enfants. De lundi au vendredi, j'accompagnerai mes enfants au garderie [...] (Jeanne).
\end{abstract}

Raphael et Jeanne ${ }^{8}$ font des erreurs, mais ils maitrisent la conjugaison au futur simple des verbes courants qui a fait l'objet d'un entraînement intensif. Leurs textes sont intégralement écrits au futur.

Je vais rentrer en Chone? Je vais marier avant 28 ans $^{-}$....]. Je vais essayer de chercher un bon job[...]. Je vais voyager dans beaucoup de pays surtout en l'europe (Corinne).

Corinne a tout écrit au futur périphrastique. Elle vise probablement la correction des phrases car elle ne maitrise pas très bien la conjugaison du futur. "Avant 28 ans" lui permet d'ancrer son discours dans un temps psychologiquement relativement proche (moins des 10 ans de la consigne pour elle).
Je me suis mariée il y a 3 ans. J'aurai un garçon dans 4 mois [...]. Ces derniers jours, j'étais occupée. Je suis en train de décorer la chambre de mon fils. Je lui donnerai une chambre très mignonne. Demain, je vais rentrer voir mes parents avec mon mari. Ils sont en très bonne santé. Ils organiseront une fête de voisins au moins de juin. Ce sera gégniale (Weijin).

La production est correcte, mais la distinction futur proche/lointain dans le temps est sous-jacente : "dans 4 mois" vs "demain" ; pourquoi pas "demain, j'irai voir mes parents" (commentaire d'un étudiant français qui a participé à l'analyse et l'évaluation des productions)? Wenjin ajoute: La grammaire est la plus difficile. Souvent je préfère utiliser "aller + verbe pour éviter la conjugaision".
50 ans, je crois que le monde deviendrait très industrialisé. Les gens auraient recours aux machines et appareils automatiques et intelligents pour la vie quotidienne. Donc ce serait possible que nous ne fassions pas les choses nous-mêmes, car ils pourraient les faire pour les humains. Et aussi, les humains vivrions plus longtemps qu'aujourd'hui (Madeleine).

41 Madeleine maîtrise la conjugaison du conditionnel, elle l'emploie parce qu'elle fait des hypothèses à long terme. Mais la concordance, sur le plan textuel, n'est pas correcte car la première phrase commence par un verbe de croyance qui impliquerait ensuite un futur exprimant la probabilité de réalisation.

Le monde dans 10 ans: la planète deviendrait encore plus chaud. Les icebergs d'artique fondront. L'émission de carburant serait diminuée. Les voitures électriques et hybrides seront largement utilisés. Le robot paraitrait au quotidien (anonyme).

L'étudiant applique la règle qui distingue le futur probable/certain du conditionnel à valeur hypothétique. Il commente : "Au début, les conjugaisons me posent des difficultés. Mais au fur et à mesure de l'étude, je trouve que les valeurs des temps et modes utilisés 
sont le plus difficile". La morphologie est acquise, ce n'est pas le cas pour l'utilisation correcte de ces formes en discours.
Je préfererai revenir en Chone au moins, c'est mon idée actuelle. J'aurai un très bon travail [...] Je voudrais me marier avec une fille maigre et pas très petite. Elle serait un peu traditionnelle, mais un peu aussi ouverte (Alex).

Toute la partie projeté dans le futur est de l'ordre du certain (travail, maison, voyages...), c'est d'ailleurs le but explicite des jeunes Chinois modernes qui s'expatrient pour faire des études à l'étranger. En revanche, la fin du texte exprime un souhait, qui pourra ou non se réaliser, vu le ressenti de la difficulté à trouver l'épouse idéale. Cette différence futur/conditionnel est justifiée par des étudiants de niveau intermédiaire A2/B1: "Je voudrais" est l'expression prototypique du souhait, ce qui n'est pas le cas de "préférer" ou "aimer", ces verbes étant utilisés dans des contextes d'actes de langage différents dans les manuels de langue, le premier pour la demande polie, les deux autres pour l'expression affirmée des goûts.

\begin{abstract}
A la fin de l'année universitaire, je devrai préparer pas mal de dossier et d'examens, ainsi que le mémoire. Et après tout cela, je ne rentrerai pas en Chine, car le billet d'avion coûte trop cher. Je compte travailler un peu pour les vacances d'été, comme serveuse dans un restaurant. Donc je pourrai gagner un peu d'argent dans ce cas là pour payer l'année prochaine. Et aussi je crois que je pourrai pratiquer plus le français si je reste en France cet été au lieu de rentrer en Chine et parler chinois tous les jours.

Marine - Salut, ça va ? - Oui, oui ça va et toi ? - Ca va bien, tu sais, je partirai pour Paris dans trois jours. Ah, super, c'est pour quoi faire? - En fait, j'ai reçu la confirmation de stage avant-hier, alors je ferai un stage dans une école de langue pour trois mois à Paris, à Alliance française de Paris (Marine).
\end{abstract}

Marine possède un bon niveau de français, le texte est correct à l'écrit (sauf la confusion pour/pendant qui est un calque de l'anglais). En revanche, dans le dialogue, elle utilise le futur, alors qu'on s'attendrait à un meilleur ancrage dans l'énonciation. En effet, les Français utiliseraient plutôt le futur périphrastique ou le présent dans ce contexte. Même si Marine connaît quelques "petits mots" de la conversation ordinaire, il semble qu'elle n'ait pas encore pris conscience et/ou acquis l'emploi du présent et du futur périphrastique à l'oral. Le niveau $\mathrm{B} 2 / \mathrm{C} 1$ est acquis à l'écrit mais des progrès sont à faire pour l'oral de la conversation ordinaire qui n'est pas beaucoup étudié en Chine.

Après mes études universitaires en France, je vais rentrer en Chine [...] c'est-à-dire que je travaillerai comme un enseignant de français à l'université [...]. Si c'est possible, j'aimerai continuer à faire mes études en France. "Peut-être, je choisirai une formation commerce international cette fois. Je vais essayer de postuler la grande école comme " Ecole Audencia » (Daniel).

Le texte au futur est encadré par deux futurs périphrastiques, qui renvoient à une double interprétation temporelle et modale comme en chinois, plutôt qu'à un ancrage dans l'énonciation : le retour en Chine puis à nouveau en France. Les autres phrases, au futur, montrent que l'étudiant connaît la conjugaison du futur et applique la règle de l'utilisation du futur pour projeter les réalisations probables de sa vie à venir, $\mathrm{y}$ compris pour le verbe "aimer" dans la structure complexe apprise "Si + présent, principale au futur". 
Je voudrai avoir un bon travail entre 20 et 35 ans. C'est une période d'or. Je souhaite tout mettre en cuvre pour atteindre mes objectifs. Ensuite, entre 30 et 40 ans, je pense que c'est le bon moment pour fonder une famille. Il vaut mieux que j'aie une famille stable. Et après 50 ans, j'aimerai avoir une belle maison et assez d'argent. Je pourrai faire beaucoup de voyages avec ma famille. J'espère ne pas devoir m’inquiéter pour l'argent (Yichen). utilisé, mais les emplois sont erronés pour vouloir et aimer conjugués au futur.

\section{Synthèse des résultats}

Les problèmes constatés dans l'ensemble des récits concernent:

- Absence du présent pour parler du futur ;

- Stratégie d'évitement: choix du futur périphrastique facile à conjuguer au détriment du futur simple ;

- Absence de phrases complexe avec futur antérieur/futur simple ou structure hypothétique "si..." ;

- Représentation erronée sur la terminologie "futur proche" vs "futur simple" interprétée comme une différence proche/lointain dans le temps objectif ;

- Utilisation erronée du futur pour exprimer des souhaits, des hypothèses, des préférences (ce $n$ 'est pas un oubli de la désinence $-\mathrm{s}$ du conditionnel après confirmation en entretien mais le choix du temps futur simple pour des verbes exprimant un souhait, une préférence...) ;

- Erreurs sur la concordance des temps avec les verbes de croyance;

- Mélange des formes et erreurs de conjugaison chez les étudiants les moins avancés (A2-B1 dans le corpus), peu d'erreurs de conjugaison au niveau B2.

\section{Conclusion}

Suite à ces observations concernant l'expression du futur par les étudiants chinois, il serait intéressant de travailler sur la représentation du temps d'un point de vue interculturel (cf. supra 3 et 4 ) et, d'un point de vue linguistique (cf. supra 4 et 5) sur l'utilisation actuelle des temps en français : présent, futur périphrastique, futur simple et conditionnel. Un autre point à développer serait la phrase complexe ("après avoir..." ou "quand j'aurai fait..."), le chinois de la conversation ordinaire préférant les phrases simples juxtaposées, ce que reproduisent les étudiants. Ils ont par ailleurs tendance à formuler leurs idées en chinois puis à les traduire en français, ce qui est confirmé par quelques-uns.

Les manuels à tendance universaliste des éditeurs français ne prennent pas en compte les difficultés spécifiques des étudiants chinois. En l'absence de connaissances préalables sur la langue et la culture chinoises, nombre de lecteurs français ont tendance à reproduire un modèle grammatical français d'enseignement de la langue qui correspond à une culture grammaticale partagée depuis le primaire et à analyser les erreurs en termes de calques entre le chinois et le français, sans prise en compte de l'anglais qui interfère forcément dans l'acquisition postérieure du français.

Je préconise des activités métalinguistiques de type interlinguistique chinois-anglaisfrançais et intralinguistique français à la place du par cœur - recette, fondé sur la 
répétition et la reproduction de modèles dans le mode d'enseignement en Chine. Les recherches en acquisition montrent en effet que les apprenants s'appuient sur leurs différentes compétences langagières, conscientes ou inconscientes, pour construire une compétence dans une autre langue, en particulier par la réflexion sur le fonctionnement des langues qui favorise l'acquisition des langues et a, en retour, un effet positif sur les langues précédemment apprises (Zarate \& al., 2009 ; Castelotti \& Moore, 2008 ; Vasseur, 2005 ; Gajo, 2001).

La représentation que l'anglais et le français sont deux langues proches, due à la transparence lexicale et à la proximité géographique, n'est pas non plus une évidence et mériterait d'être réexaminée dans une perspective contrastive et didactique (Cuet, 2009).

\section{BIBLIOGRAPHIE}

Ahrens K. \& Huang C-R. (2002). "Time passing is motion". Language and linguistics, vol. 3, $\mathrm{n}^{\circ}$ 3. pp. 491-519. http://ntur.lib.ntu.edu.tw/bitstream/246246/65246/1/09.pdf

Boroditsky L., Fuhrman 0. \& McCormick K. (2010). "Do English and Mandarin speakers think about time differently?". http://psych.stanford.edu/ lera/papers/mandarin-time-2010.pdf

Moore D. \& Castellotti V. (dir.). (2008). La compétence plurilingue : regards francophones. Fribourg : Peter Lang (coll. Transversales).

Cheng, A. (1997). Histoire de la pensée chinoise. Paris : Éditions du Seuil.

Cuet, C., (2009). "Enseignement plurilingue en Chine. Une voie pour la recherche ?". In Forlot (dir.). L'anglais et le plurilinguisme. Pour une didactique des contacts et des passerelles linguistiques (postface V. Castellotti). Paris : L'harmattan (coll. Espaces discursifs). Chap. 6. pp. 117-139.

Cuet, C. \& Marguerie, A. (2008). "La notion d'espace-temps. Quelles représentations ? Quelles implications en didactique du FLE avec un public chinois ?". In Marillaud \& Gauthier. Actes du 28ème colloque d'Albi "Langages et signification: Temps et temporalité".

Cuet, C. (2011). "Enseigner le français en Chine, méthodologies nouvelles, perspectives". In Synergies Chine $\mathrm{n}^{\circ}$ 6. pp. 95-103.

Gajo, L. (2001). Immersion, bilinguisme et interaction en classe. Paris : Editions Didier (coll. Langues et apprentissage des langues).

Gentner, D., Imai M. \& Boroditsky L. (2002). "As time goes by: Evidence for two systems in processing space - time metaphors". Language and cognitive processes, vol. 17 (5). pp. 537-565. http://www-psych.stanford.edu/ lera/papers/timegoesby.pdf

Granet, M. (1999). La pensée chinoise. Paris : Albin Michel.

Hall, E.-T. (1984). La dimension cachée. Paris : Seuil.

Jullien, F. (2001). Du "temps". Eléments d'une philosophie du vivre. Paris : Grasset.

Lakoff G., Johnson M. (1985). Les métaphores dans la vie quotidienne. Paris: Editions de Minuit (coll. Propositions). 
Levinson, S. (2003). Space in language and cognition: explorations in cognitive diversity. Cambridge: Cambridge University Press. Compte-rendu par Fortis http://www.ddl.ish-lyon.cnrs.fr/ trajectoire/23us23efd5ps/CRsLectures/Levinson2003CR_Fortis.pdf

$\mathrm{Pu}, \mathrm{Z}$. (2003). Politesse en situation de communication sino-française : Malentendu et compréhension. Paris : L'harmattan.

Roche, P. (2007). Grammaire active du chinois. Paris : Larousse.

Vasseur, M-T. (2005). Rencontres de langues : Question(s) d'interaction. Paris : Editions Didier (coll. Langues et apprentissage des langues.

Vendler, Z. (1957). "Verbs and Times". Repris dans Vendler (1967) Linguistics in Philosophy, Ithaca: Cornell University Press, pp. 97-121. Commenté par Recanati (1994) http://hal.archivesouvertes.fr/do,cs/00/08/50/94/DOC/Vendler.doc

Xu, D. (2010). Initiation à la syntaxe chinoise. Paris : Langues INALCO (Coll. L'asiathèque).

Xu, Y. (2010). "Différences méthodologiques entre les manuels français et chinois de FLE sous l'angle de l'organisation structurelle du contenu". In Synergies Chine n 5. pp. 89-98.

Zarate, G. \& al. (2009). Précis de plurilinguisme et de pluriculturalisme. Paris : Éditions des archives contemporaines.

Zheng, L. \& Desjeux D. (2003). Entreprises et vie quotidienne en Chine. Paris : L'Harmattan.

\section{Sites internet}

Dictionnaire chinois - français \& Dictionnaire chinois - anglais. http://www.chine-nouvelle.com/ outils/dictionnaire.html

\section{ANNEXES}

Figure 7 - Représentation du cycle de la vie par deux étudiantes chinoises

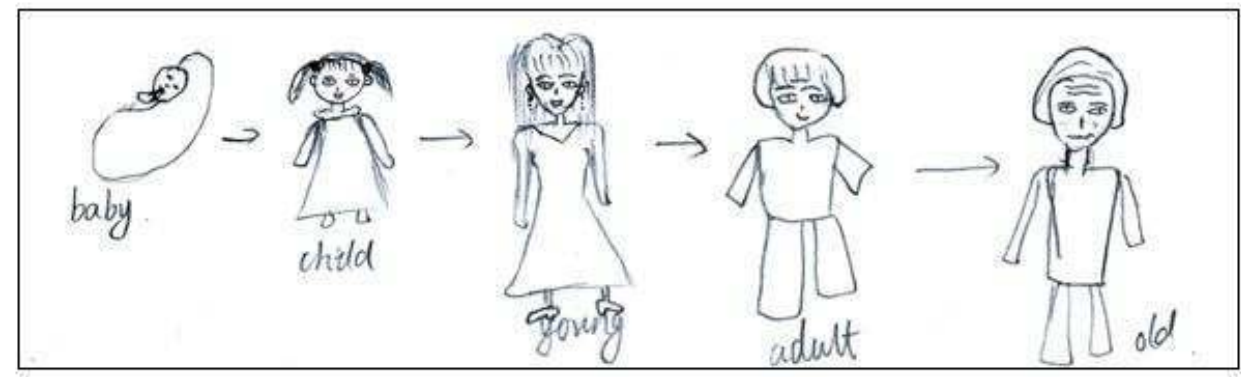




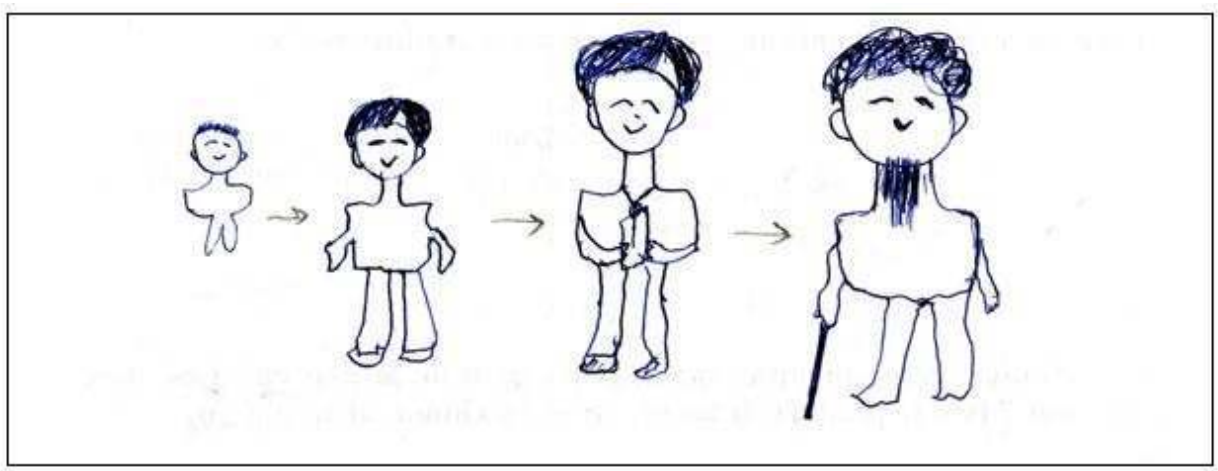

Ces deux dessins, choisis pour leur qualité esthétique parmi d'autres, témoignent d'une représentation partagée qui allie modernité et tradition. La jeune fille est belle et parée, mais à l'âge adulte, elle se conforme à la tradition, un symbole en est l'absence de pieds ; après son mariage, elle est fortement soumise au système hiérarchique traditionnel mari/ femme. Le jeune homme porte une cravate, signe de son aisance professionnelle et sociale ; quand il vieillit, la canne est le symbole de l'âge, la barbe celui de la sagesse et de la sérénité. Ces symboles incarnent le respect dû aux aînés.

\section{NOTES}

1. Ma Xiaohong, Le français (Tomes 1-4), Pékin : Edition de l'Enseignement et des Recherches des Langues étrangères; Un autre manuel très utilisé est "Reflets" édité par Hachette à la fin des années 1980, qui propose une version "à la chinoise". Voir Xu Y. (2010) pour une étude sur les manuels utilisés en Chine: "en comparant les points grammaticaux définis par les différentes leçons, nous trouvons que les manuels chinois les présentent en fonction du degré de difficulté linguistique, et les manuels français en fonction des besoins langagiers".

2. Les étudiants pouvaient répondre en français, en anglais ou en chinois au questionnaire sur les représentations concernant le temps et l'espace. Les étudiants débutants en français ont souvent préféré répondre en anglais, langue partagée avec leur enseignante française, ou en chinois, les réponses ont alors été traduites en français par une enseignante chinoise (environ 70 questionnaires exploitables).

3. Cf. Hall (1984) qui distingue les comportements monochrones et polychrones, selon que le temps est traditionnellement représenté de manière linéaire et fragmentée (progrès, évolution...) ou cyclique (répétition, permanence...).

4. Même valeurs symboliques en occident: on est "en haut" ou "en bas" de l'échelle sociale, la "haute bourgeoisie", les "bas-fonds" de la société, l'École normale "supérieure", etc.

5. Il existe bien entendu des différences en fonction des régions, des langues, des dialectes, du niveau d'éducation et de l'âge. En situation de communication authentique en Chine, en l'absence de pointage ou de carte tournée dans le bon sens, un Français aurait bien des difficultés à se repérer en observant le soleil!

6. Avec les verbes atéliques (états et activités), c'est "pendant" qui indique la durée (l'intervalle de temps) ; "j'ai un travail à finir/faire pour demain" : dans ce cas "pour" est possible car "finir" et "faire" sont des verbes téliques marquant l'achèvement/l'accomplissement (Vendler, 1957). "Pour" indique une orientation vers la localisation de la cible "il part pour Paris" (spatiale), "il a réservé une table pour samedi" (temporelle), "il a de l'admiration pour Jean" (affective), etc. 
7. Un temps pour le présent, deux temps pour le passé (imparfait/passé composé correspondant à inaccompli et duratif/accompli et non duratif), deux temps pour le futur (futur périphrastique proche/futur simple - lointain).

8. Les étudiants chinois ont l'habitude de se choisir un prénom français quand ils commencent à étudier le français. Nous avons repris le prénom, français ou chinois, qui apparait sur les productions.

\section{RÉSUMÉS}

La culture s'inscrit dans la langue, le temps (time) est représenté différemment en chinois et en français. En chinois, le futur, qu'il soit objectivement proche ou lointain, qu'il soit probable, certain ou hypothétique, est exprimé par des modalités, ce qui est aussi le cas en anglais. Des adverbes et des particules apportent le complément d'information temporel et aspectuel. Le système verbal français est difficile à acquérir, d'une part à cause de la complexité de la conjugaison, d'autre part à cause des multiples contextes d'utilisation des formes verbales. Le corpus étudié montre que les Chinois de niveau B2 ont encore beaucoup de difficultés à comprendre le fonctionnement temporel, modal et aspectuel du système français ainsi que celui des prépositions spatiales et temporelles. La forme traditionnelle de l'enseignement et les manuels de FLE (Français Langue Étrangère) sont en partie responsables des erreurs récurrentes observées.

Culture is embedded in language, thus time is represented differently in Chinese and in French. In Chinese, the future, be it objectively near or far, probable, certain or hypothetical, is expressed by modals, which is also the case in English. Adverbs and adverbial particles provide complementary aspectual-temporal information. The French system of verbs is difficult to acquire, partly due to the complexity of conjugation, and partly because of the wide variety of contexts in which each verb form is used. The corpus studied shows that Chinese learners at a B2 level still have difficulty understanding the temporal, modal and aspectual functions of French as well as the use of spatial and temporal prepositions. Traditional styles of teaching and French language manuals may be partly responsible for the recurrent errors observed.

\section{INDEX}

Mots-clés : temps, futur, français, chinois, acquisition

Keywords : time, tense, future, French, Chinese, acquisition

\section{AUTEUR}

\section{CHRISTINE CUET}

Christine Cuet est maître de conférences à l'université de Nantes. Sa recherche porte sur les représentations translinguistiques et transculturelles, l'acquisition du français par les Chinois, le plurilinguisme, l'enseignement précoce, les pratiques artistiques en classe de langue, 
l'expression multimodale.

Courriel : christine.cuet@sfr.fr

Adresse : 57, rue Charles Monselet, 44000 Nantes. LLING (Laboratoire de Linguistique de Nantes), Université de Nantes, Chemin de la Censive du Tertre, BP 81227, 44312 Nantes cedex 3. Membre du Comité de pilotage de Pluri-l, projet régional des Pays-de-la-Loire (CREN : 2009-2014) http:// www.projetpluri-l.org/ 